\title{
A SURVEY ON HANDWRITTEN CHARACTER RECOGNITION (HCR) TECHNIQUES FOR ENGLISH ALPHABETS
}

\author{
Manoj Sonkusare and Narendra Sahu \\ ${ }^{1}$ Dept. of Computer Sc. \& Engg.,Ujjain Engineering College,Ujjain, India \\ ${ }^{2}$ Dept. of Computer Sc. \& Engg.,Women's Polytechnic College,Indore, India
}

\begin{abstract}
Nowadays Hand written Character Recognition (HCR) is major remarkable and difficult research domain in the area of Image processing. Recognition of Handwritten English alphabets have been broadly studied in the previous years. Presently various recognition methodologies are in well-known utilized for recognition of handwritten English alphabets (character). Application domain of HCR is digital document processing such as mining information from data entry, cheque, applications for loans, credit cards, tax, health insurance forms etc. During this survey we present an outline of current research work conducted for recognition of handwritten English alphabets. In Handwritten manuscript there is no restriction on the writing technique. Handwritten alphabets are complicated to recognize because of miscellaneous human handwriting technique, difference in size and shape of letters, angle. A variety of recognition methodologies for handwritten English alphabets are conferred here alongside with their performance.
\end{abstract}

\section{KEYWORDS}

Handwritten Character Recognition (HCR), features extraction, Optical Character Recognition (OCR), classifiers, Pre-Processing

\section{INTRODUCTION}

The Optical Character Recognition (OCR) is a broad domain of research in Soft Computing, artificial intelligence (AI), pattern recognition (PR) and computer vision. OCR is a general technique of handwritten texts or digitizing pictures of printed so that they could be electronically amended, stored and searched more efficiently and correctly. While study of decades and enhancement in this region, machines are still nowhere near to human's analysis capabilities [1]. The objective of an OCR method is recognition of manuscript (same as humans) in a difficult article. OCR methods are majorly categorized in two types, first online text recognition and second offline text recognition [1]. Offline OCR is considered in two sub categories first is Typed text and second is Handwritten Text, figure 1 shows classification of OCR.

In online text recognition, words and characters are recognized at run time as quickly as they are written, and consequently, have temporal information. Online methods gain the position of the pen as a function of time straight from the interface. This is typically done through pen-based interfaces somewhere the author writes with an individual pen on an electronic tablet. Dynamic knowledge, which is usually available for online text recognitions, is total strokes, set of strokes, approach of stroke, and velocity of writing within each stroke. This precious information helps in recognition of articles and often guides to enhance performing methods contrasted to offline

DOI : $10.5121 /$ avc. 2016.3101 
recognition. Several applications of online optical recognition are in PDAs, Tablet computers and smart phones. The benefit of online recognition methods over offline methods is interactivity, acceptation of author to digitizer (or vice versa), available temporal information and fewer prone noise. The drawback is that the entire article is not obtainable for processing and then, information requires to be processed dynamically [5] [6].

An Offline text recognition method processes a static representation of an article. Recognition offline text is partitioned in two sub categories of Typed and Handwritten articles. In both sub categories, an image of the article gained from a scanner or camera is processed. Clearly, due to the variation of styles in handwriting and un-benchmarked nature of handwritings, the problem of offline handwriting recognition is the main exigent complexity in OCR and it usually requires language specific techniques. On the another hand, OCR of typed articles are extremely much in demand for practical applications such as historical article analysis, official note and article processing, and vehicle plate recognition. OCR of typed document for English alphabets has become one of the major successful applications of technology in pattern recognition (PR) and artificial intelligence (AI). Almost all of the present research in this area is to covenant with extremely complex articles, noisy and twisted articles, as fine as improving recognition rates for texts [7] [8].

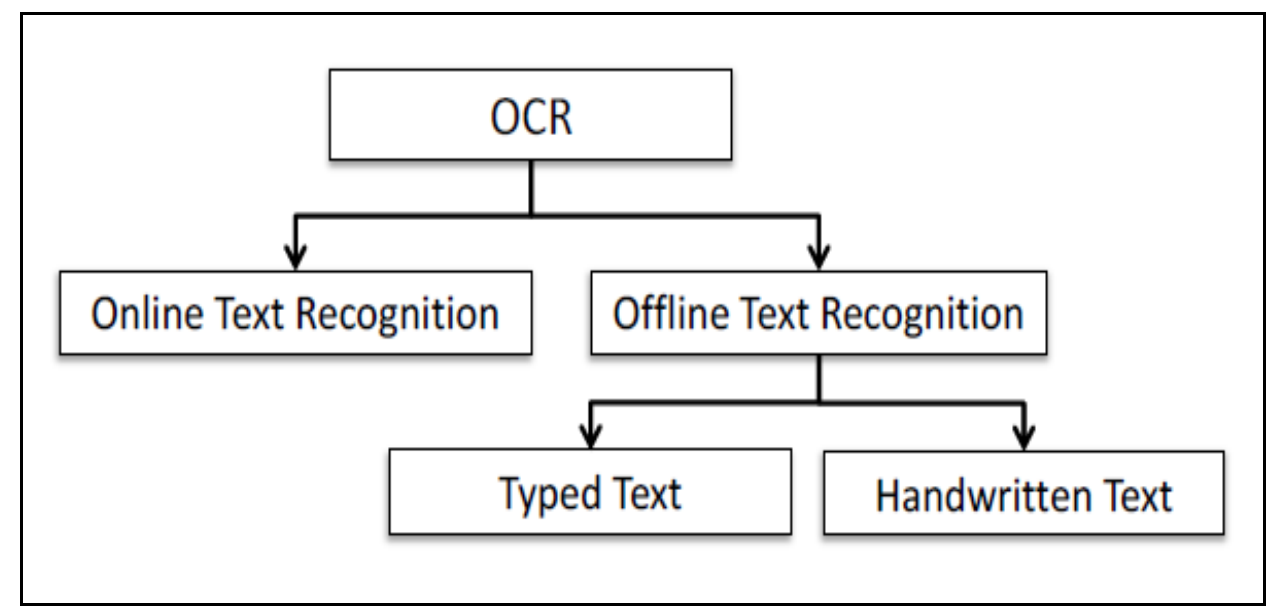

Figure 1: Optical Character Recognition Classifications

Handwriting recognition is the major interesting and complicated study domain in fields of image processing and pattern recognition in current years [2] [3]. It contributes immensely to the sequence of a computerization technique and could enhance the interface among human and machine in so many purposes. Various study attempts have been centre of attention on innovative methods and schemes that would decrease the processing moment while offering maximum recognition precision [4].

Rest of the paper id organized as follow: in section 2 handwriting recognition is described in detail, followed by related work done in the field of HCR in section 3, section 4 gives briefly problems in current HCR system and finally we concluded paper in section 5.

\section{HANDWRITING RECOGNITION}

The primary significant way in some recognition of handwritten English alphabets scheme is pre processing succeeded by segmentation procedure and feature extraction procedure as represents in figure: 2 . The Pre processing includes the phases that are necessary to construct the raw image 
in a figure which is appropriate for segmentation [9]. In the segmentation, the raw image is segmented in single characters and after that, every character is restructured in $\mathrm{m} \mathrm{x} \mathrm{n}$ pixels to the training network.

The choice of suitable feature extraction method is perhaps the particular mainly significant issue in achieving high recognition percentage. Various methods of feature extraction for identification of alphabet have been projected in the research work of [10]. The frequently used feature extraction techniques are Contour profiles, Deformable templates, Fourier descriptors, Gabor features, Gradient feature, Graph description, Geometric moment invariants, Template matching, Unitary Image transforms, Projection Histograms, Zoning, Zernike Moments and Spline curve approximation.

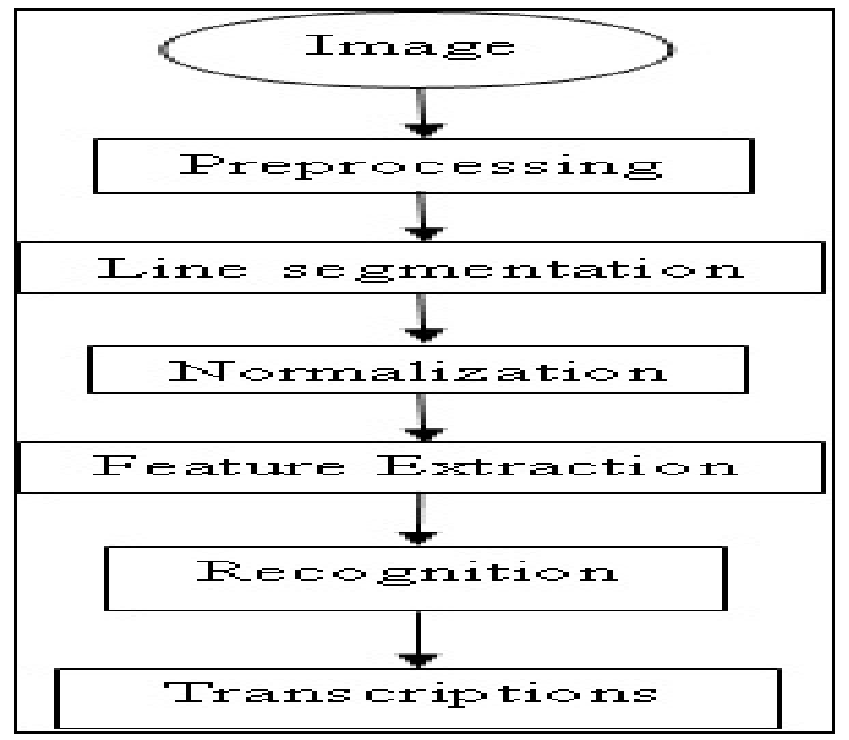

\subsection{Pre-processing}

Figure 2: Handwritten recognition system overview

The pre processing is an actions chain which is carried out on the scanned input image. It basically improves the image rendering it appropriate for segmentation. In pre processing of an image so many operations are executes on it which are shown in fig.3. 


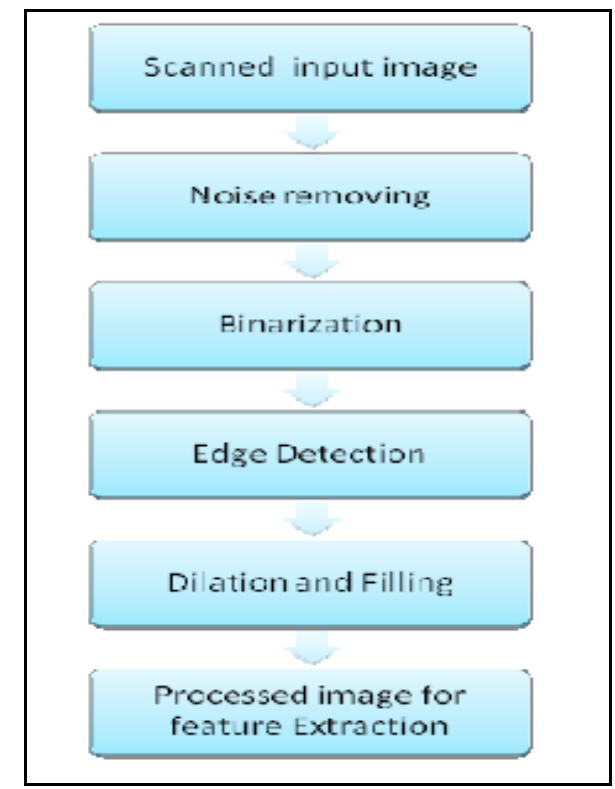

Figure 3: Pre-processing of handwritten character

\subsubsection{Removal of Noise}

The pre processing stage enhances the superiority of the raw image and puts the data of significance. It is also acknowledged as pixel level or low level processing, which is arranged on the detained image to manage it for additional analysis. Skew correction of an image is executes in pre processing phase to accurate the article text lines, threshold to switch a gray scale or color image into a binary image, and decrease of noise to decrease extraneous data as shown in Figure 4.

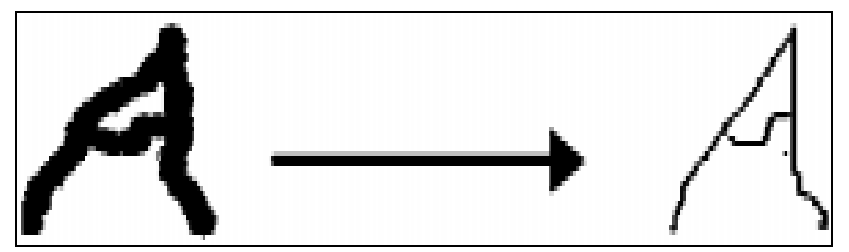

Figure 4: Pre processing phase results, including noise removal and skew correction

Scanned documents can be contaminated with dust, spots, dots, or lines, which are classified as noise that affect the recognition results to a large degree. Consecutively to construct it appropriate for additional processing, a scanned article image has to be freed from any existing noise. Image enhancement methods are applied to improve the image being viewed by the machine or human. Smoothing and non linear operations, such as morphological operations, are utilized for noise decrease.

\subsubsection{Skew Correction}

Skew correction is used to correct the text line in scanned document images if they are not horizontally aligned during the scanning process. The skew correction can be applied on the document or line level. In the preprocessing step, a global skew correction is applied on the document level. 


\subsection{Line Segmentation}

The phase of segmentation in pre processing is an image in structure of sequence of alphabets is decayed into sub images of single character. The input image which was pre processed is fragmented into separated alphabets by conveying a number to every alphabet utilizing a labelling procedure. This labelling offers knowledge concerning to number of characters in the image. Every single character is homogeneously restructured into $90 * 60$ pixels for categorization and recognition stage.

\subsection{Normalization}

Once the lines are extracted from the document image, the normalization step is performed before extracting the features. The major purpose of the normalization phase is to eliminate the differences that would otherwise complicate the categorization and decrease the recognition rate of the similar character or word across different authors. The most general basis of variability in handwritten alphabet images is incline and text volume.

\subsubsection{Slant Correction}

Slant correction is applied to correct the inclination in the writing style. By applying a shear transformation, the writings slant is transformed into its upright position. A shear transformation is applied in many directions. For each direction, the transformed image data are added with pixel values of the same image that are vertically shifted by distance $d$ and $-d$ shown in fig 5

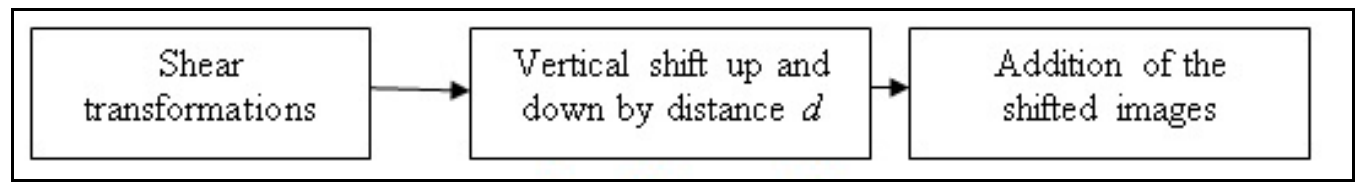

\subsection{Features Extraction}

Figure 5: Slant correction

Features play an important role in handwriting recognition systems. Their main goal is to increase the recognition rate by effectively representing the data. Feature extractions covenants with extracting mainly with the necessary information from image pixels depending upon the difficulty to be resolved and the utilized data. In handwritten alphabet recognition systems, two main feature extraction approaches are used holistic and analytic. In holistic recognition, each word is considered to be a class and is recognized as whole word. On the other hand, the analytic recognition approach is based on character segmentation-free recognition

Based on [11], features used in holistic offline recognition systems are categorized into high, middle, and low levels. The high level removes the features from the entire phrase image, the middle level removes features from the letters, and the low level removes features from the sub letters. Various types of holistic features are used, such as the following [11]:

- Raw intensity features

- Statistics of local regions (mean, variance, and other higher order moments of the intensity)

- Features describing the connectivity of strokes including the directional features and Gabor features

- Concavity, run length, and other structure features. 
- Quantity of ascenders and descenders,

- Power projection profile

In analytical recognition schemes, the image is signifying like a chain of characteristics permits an influential employ the hidden Markov models (HMM), which is mostly utilized for chain modeling. Thus, rather than extracting characteristics from the entire image, a sliding window is shifted over the image from left to right. At every position $\mathrm{n}$, a characteristic vector $\mathrm{fn}$ is calculated from just the pixels within the sliding window, as represent in Figure 6.

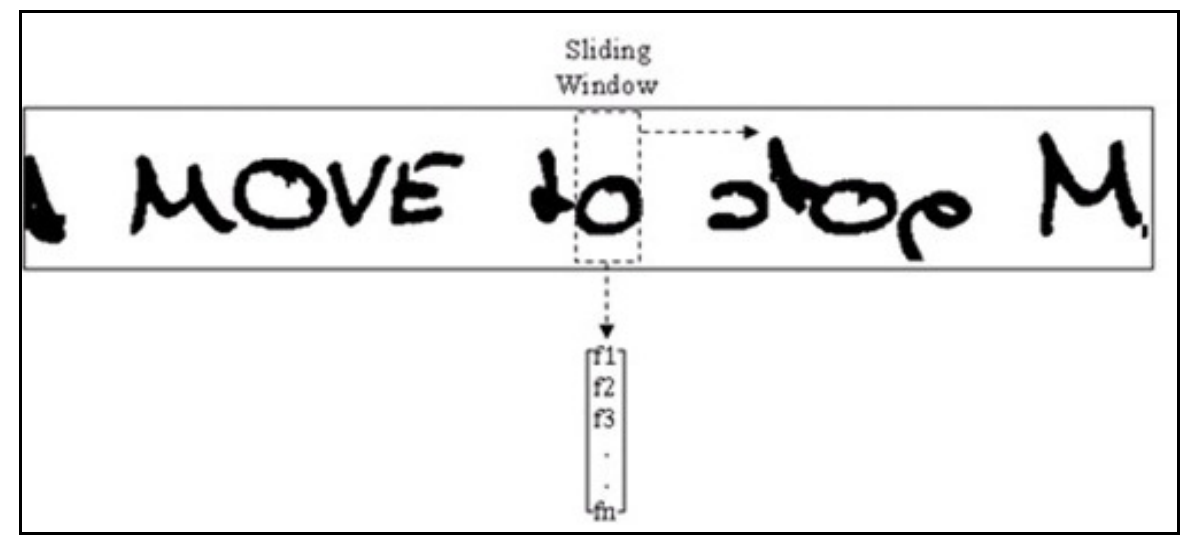

Figure 6: Feature extraction using a sliding window.

The sliding window scheme defends the left to right scripting nature of the article as fine as the inconsistent alphabet extent characteristics.

Two major sliding window scheme characteristics are based on the size of the sliding window are as follows:

- Profile Characteristics: this characteristic was projected by writers of [12] and illustrated high precision for English handwritten alphabet recognition. They utilized a sliding window of one pixel size. The Profile characteristics consist of 9 geometrical characteristics. Characteristics from 1 to 3 are the weight of the window, its centre of gravity, and the second order moment of the window. Characteristics 4 and 5 describe the location of the upper and the lower curve in the window. Characteristics 6 and 7 provide the direction of the upper and the lower curve in the window by the incline of the curve at the windows location. For characteristic 8 , the number of black white changes in the vertical direction is utilized. Finally, characteristic 9 gives the average grey value among the upper and lower curves [12].

- Window Characteristics: characteristics where the size of the sliding window is above one pixel. The most admired characteristics were projected by writers [13] and [14] and are demoted to as window characteristics. In [13] characteristics, the sliding window is divide into $4 \times 4$ cells recognized as bins, and the pixel calculates in every bin are measured to be a characteristic, resulting in a 16 dimensional characteristic vector.

\subsection{Recognition}

Recognition of handwritten English alphabets is extremely difficult problem. The English alphabets might be scripted in various size, direction, width, arrangement and measurement. This might provide endless differences. The potential of neural network (NN) to simplify and insensitive to the misplaced information might be extremely helpful in identifying handwritten English alphabets. 
The categorization phase is the assessment building element of a recognition scheme and it utilizes the characteristics removed in the earlier phase. The feature vector is signified as $\mathrm{X}$ where $X=(f 1, f 2, \ldots . ., f d)$ where $f$ signifies features and $d$ is the no. features removed from English alphabets. On the basis of similarity of feature vector English alphabets are powerfully categorized into suitable category and acknowledged. Classifiers works on basis of two types of learning techniques:

- Supervised learning: In supervised learning training information with accurate specification of category is pertain to train a novel model. This novel model is utilized to test information for appropriate categorization. Training information comprises both the input and the required results. The novel model suffers learning procedure and on this basis it learning \& it classifies test information. For example: HMM, SVM etc.

- Unsupervised learning: Unsupervised learning technique is not granted training data. It doesn't need learning. The technique categorizes test information on basis of statistical characterstics and by their spatial alliance and considers their closest neighbour. For example: $\mathrm{k}$ means, Clustering, etc

\subsection{Transcription}

In this phase of pre processing precision of identification is additional amplified by linking dictionary to the scheme in order to achieve Syntax analysis and semantic analysis sort of higher level conceptions, which is pertains to verify the identified alphabet. This phase is not obligatory in HCR scheme.

\section{RELATED WORK}

Handwritten alphabets recognition system's precision of any image relay upon the sensitivity of the choice of features and category of classifier utilized. Therefore, so many feature removal and categorization techniques could be establish in the literature. Following research work execute handwritten alphabets recognition of English phrases.

To recognize cursive alphabet Author of [15] states Holistic technique. This technique utilized in signifying phrase via different transformation phases like contours, features, letters, phrase and points. Feature vector is exclusively created from image to utilizing arithmetical trusts among character and features, partially calculated characters are identified by evaluating through lexicon. Lexicon comprises of only 130 words, thus restricted number of words are identified.

For recognization of characters classifiers aren't used, a ranking is provided to every section which is detached with initial segmentation process utilizing character hypothesis and they identified on the basis of highest value of ranking [16]. This research work uses holistic technique to recognition of cursive alphabet. They remove some features from the frame of character. The vector of feature is produced from the boundary data of characters which comprises position of boundary connected to 4 situation lines, its curve node degree event to the boundary etc. 10 dimensional vectors of feature is produced. HMM for every character of alphabets is framed and by integration of these HMMs, HMM for every thesaurus alphabet is framed. Restricted ranged thesaurus is utilized. HMM is skilled via Baum Welch method and Viterbi algorithm is used for recognization.

To recognize both cursive and isolated handwritten alphabets the writer of [17] had executed recognization technique by applying HMM. Hybrid technique is utilized to make best use of the supremacy of HMM. To identify of alphabets features it utilized averages of black run in every scan line. Alphabet image is inspected in 4 various directions to mine features from it. Average of 
each direction rises for a sparse directional frame of the alphabet. The isolated compactness from left to right HMM method is utilized for identification. To recognize cursive alphabets writers utilized segmentation scheme for recognization. Features are supplied to the upper order of HMM and at last segmentation route are verified. To utilize graph search technique accurate segmentation points are created which is smallest route with smallest cost. The possibilities of surveillance chain of HMM are utilized for recognization.

To identify handwritten English cursive alphabets utilizing segmentation technique research work is presented in [18]. This research work states the evaluation among two techniques. The primary scheme utilizes mixture of NN (Neural Network) and HMM (Hidden Markov Model) for recognization and in second scheme discrete HMM is utilized for recognization. In primary scheme Pre segmentation of alphabet is executed utilizing segmentation graph. Neural network calculates the possibility for every alphabet hypothesis in graph and subsequently HMM determines possibility for every alphabet in lexicon by including the possibility along every probable route in graph. In second scheme 140 geometric characteristics are mined from every section which is divided by pre segmentation. This characteristic by vector quantization (VQ) modified to single symbol and ultimately by calculating the probability for every alphabet in lexicon characters is recognized.

In research paper [19] writer stated segmentation method to identify cursive alphabets. In this scheme cursive alphabets are primary segmented into particular characters, which are than identified and merged to create meaningful phrase by comparing with thesaurus. The thesaurus utilized in this research paper exists of 26 phrases. Hence range of this research paper is limited to only those 26 words.

Authors in [20] represent recognition scheme based on Neural Network based. They utilized various neural network methods like back propagation (BP) neural network, nearest neighbour network and radial basis function (RBF) network for similar training dataset. They matched the performance of every network and enhanced the amount of neurons in hidden layer which is not dependent on starting value and finished that mixture of standard feature extraction scheme with supplying forward method stated in [21] compromises with the identification of handwritten English alphabets utilizing multi resolution scheme through Discrete Wavelet Transform (DWT) together with Euclidean Distance Metric (EDM). Distances from unidentified input prototype vector to every mean vectors are proposed by EDM. Smallest distance verifies the cluster membership of input vector of pattern. EDM offers an identification precision up to $90.77 \%$. For an instance the misclassification, the learning decision via ANN obtain enhanced recognition precision up to $95.38 \%$ via evaluating experimental results and after that produced result of recognition with Euclidean distances has additional obtain enhanced recognition precision up to $98.46 \%$.

The research paper [22] illustrates neural network based methods for segmented alphabet recognition. Two neural models along with two feature extraction techniques were examined. Directional and Transition features are utilized and matched by utilizing Back Propagation (BP) and Radial Basis Function (RBF) networks classifiers. The amount of feature vector is 100 in case of transition feature and 81 for directional feature. Research was executed by utilizing the CAS dataset, the BP (Back propagation) and RBF (Radial basis function) algorithm which utilizing two feature extraction techniques for both lower and upper case alphabets, likewise for BAC database. Directional features utilizing neural network execute enhanced than transition features.

Comparison among traditional and directional feature extraction technique is discussed in research paper [23]. 12 directional features are employed for identification of characters and digits. With the purpose of extract directional characteristic of incline feature of every pixel are 
removed from the incline costs which are plotted onto 12 way costs to the slant distance of $30^{\circ}$ among either two adjoining way costs. Vector of feature of every category is gained by getting average of feature matrix of every category. The alikeness among examine vector of feature and vector of feature of every categories is computed, examine image related to the category which has the maximum alikeness.

Handwritten character identification method of lowercase English alphabets is stated in research work of [24] via employing binarized pixels of the image as features and multi layer back propagation neural network as classifier. The alphabet image is binarized, filtered and reshaped to $15 \mathrm{X} 12$, therefore vector of feature of volume 180 is generated of every character which is provided to neural network for its learning. MSE (mean square error) be employed as weight function. The exploit of binarization characteristics with back propagation neural network classifier provides classification precision up to $85.62 \%$. It has ease of characteristics like straight pixel values are occupied.

Identification of different handwritten cursive alphabets is proposed in research work carried out in [25]. In this method various characteristics are extracted between them, two features customized edge map and multiple zoning are projected by writers. 9 characteristics are extracted and disadvantage of every characteristic is defeats by alternative. Every characteristic are independently provided as input to 9 multi layer perceptron network and outcomes this classifier are merged with every alternative by various law similar to max, mean, product and sum rule etc amongst them trained MLP merger provides highest outcome. Between projected characteristics customized edge map characteristic provides maximum outcome.

The major steps of an OCR engine are extraction of feature and classification. Numbers of extraction methods of features are combined with various classification techniques along with their result which have been used by the researchers are discussed in Table I:

Table I comparative analysis of various HCR system

\begin{tabular}{|c|c|c|c|c|}
\hline Ref. & $\begin{array}{c}\text { Publication } \\
\text { Year }\end{array}$ & $\begin{array}{c}\text { Classifier } \\
\text { Used }\end{array}$ & Features & Accuracy \\
\hline [19] & 2011 & $\begin{array}{l}\text { NN \& } \\
\text { SVM }\end{array}$ & Fourier Descriptors & $62.93 \%$ \\
\hline$[24]$ & 2013 & NN & $\begin{array}{l}\text { Character image reshaped to } 15 \mathrm{X} 12 \\
\text { volume, } \\
\text { feature vector of volume } 180 \text { is produced }\end{array}$ & $85.62 \%$ \\
\hline$[21]$ & 2012 & ANN & Discrete Wavelet Transform (DWT) & $98.46 \%$ \\
\hline$[16]$ & 1995 & HMM & $\begin{array}{l}\text { Location, curve of edge } \& \text { percentage of } \\
\text { pixels lying on the edge }\end{array}$ & $98 \%$ \\
\hline$[20]$ & 2012 & NN & $\begin{array}{l}\text { Character reshaped into } 30 \times 20 \text { pixels } \\
\text { gets like feature }\end{array}$ & $94.15 \%$ \\
\hline$[22]$ & 2003 & $\begin{array}{l}\text { BP and } \\
\text { RBF } \\
\text { networks }\end{array}$ & Directional and Transition features & $85.48 \%$ \\
\hline
\end{tabular}


Advances in Vision Computing: An International Journal (AVC) Vol.3, No.1, March 2016

\begin{tabular}{|c|c|c|c|c|}
\hline$[17]$ & 1998 & HMM & $\begin{array}{l}\text { medians of black run in vertical, } \\
\text { horizontal and diagonal scrutinize lines } \\
\text { for obtaining directional skeleton }\end{array}$ & $65 \%$ \\
\hline [15] & 1989 & $\begin{array}{l}\text { Word } \\
\text { formation } \\
\text { Using letter } \\
\text { hypothesis }\end{array}$ & $\begin{array}{l}\text { Contour tracing, event construction, } \\
\text { Letter hypothesis and word hypothesis }\end{array}$ & $77 \%$ \\
\hline [25] & 2010 & MLP & Customized Edge Maps and Multi zoning. & $91.39 \%$ \\
\hline [23] & 2013 & $\begin{array}{l}\text { directional } \\
\text { Pattern } \\
\text { matching }\end{array}$ & 12 directional features & $88.29 \%$ \\
\hline [18] & 2001 & $\begin{array}{l}\mathrm{HMM} \& \\
\text { NN }\end{array}$ & $\begin{array}{l}140 \text { geometrical features of every pre } \\
\text { segmented frames }\end{array}$ & $96.1 \%$ \\
\hline
\end{tabular}

\section{Problem in HCR}

Character recognition $(\mathrm{CR})$ is a procedure of machine simulation of human interpretation. It is a skill of acquiring, cleaning, recognizing and segmenting characters from an image. This procedure translates the image beneath consideration into a revisable design. Procedure of handwritten characters usually is favourable in numerous fields like signature and courtesy amount of bank cheque, information filled in tax forms, reading zip codes on envelopes etc. Numerous techniques for extraction of features of characters are helpful technique as it focuses on local characteristics of the characters. Thus it assists to gain exhaustive knowledge of alphabets therefore enhancing the procedure of recognizing characters. Task of character recognition (CR) is serious in creating a paperless situation by converting older handwritten articles into electronic collection.

HCR gives extremely to the growth of automation procedure and thus improving the communication among human and machine. It isn't easy job to build a software program to achieve $100 \%$ precision for identification of handwritten English alphabets just as still though humans too create errors to identify alphabets correctly. Handwritten characters differ depending upon the writer (similar or diverse). So there is forever a necessity to enlarge a proficient handwritten recognition system. HCR has some potential applications which generate the requirement for developing such schemes in an advanced manner. It facilitates to decrease the space need to save the data and creates it flexible to utilize. 


\section{Conclusion}

The major approaches utilized in the domain of recognition of handwritten English alphabets through the previous decade have been analyzed in this survey paper. Various pre processing, segmentation methods, feature extraction process, classification techniques are discussed in detail. Although, different techniques for treating the difficulty of handwritten English alphabets have developed in previous decades, still a lot of research is needed so that a practical software solution could be made accessible. The existing HCR for handwritten has very less precision. We require a proficient solution to resolve this difficulty so that overall performance can be amplified.

\section{REFERENCES}

[1] Eikvil, Line. "Optical character recognition." citeseer. ist. psu. Edu / 142042. Html (1993)

[2] Mori S, Suen CY, Yamamoto K." Historical review of OCR research and development” Proceedings of the IEEE. 1992 Jul;80 (7):1029-58.

[3] Impedovo S, Ottaviano L, Occhinegro S. “Optical character recognition-a survey". International Journal of Pattern Recognition and Artificial Intelligence. 1991 Jun; 5 (01n02):1-24.

[4] Govindan, V. K., and A. P. Shivaprasad. "Character recognition a review."Pattern recognition 23, no. 7 (1990): 671-683.

[5] Plamondon, Réjean, and Sargur N. Srihari. "Online and off-line handwriting recognition: a comprehensive survey." Pattern Analysis and Machine Intelligence, IEEE Transactions on 22, no. 1 (2000): 63-84.

[6] Arica, Nafiz, and Fatos T. Yarman-Vural. "An overview of character recognition focused on off-line handwriting." Systems, Man, and Cybernetics, Part C: Applications and Reviews, IEEE Transactions on 31, no. 2 (2001): 216-233.

[7] Bhattacharya, Ujjwal, and Bidyut B. Chaudhuri. "Handwritten numeral databases of Indian scripts and multistage recognition of mixed numerals."Pattern Analysis and Machine Intelligence, IEEE Transactions on 31, no. 3 (2009): 444-457.

[8] Pal, Umapada, Nabin Sharma, Tetsushi Wakabayashi, and Fumitaka Kimura. "Handwritten numeral recognition of six popular Indian scripts." In Document Analysis and Recognition, 2007. ICDAR 2007. Ninth International Conference on, vol. 2, pp. 749-753. IEEE, 2007.

[9] Casey, Richard G., and Eric Lecolinet. "A survey of methods and strategies in character segmentation." Pattern Analysis and Machine Intelligence, IEEE Transactions on 18, no. 7 (1996): 690-706.

[10] Anil. K. Jain and Torfinn Taxt, "Feature extraction methods for character recognition-A Survey," Pattern Recognition, vol. 29, no. 4, pp. 641-662, 1996.

[11] Cao, Huaigu, Anurag Bhardwaj, and Venu Govindaraju. "A probabilistic method for keyword retrieval in handwritten document images." Pattern Recognition 42, no. 12 (2009): 3374-3382.

[12] Marti, U-V., and Horst Bunke. "Using a statistical language model to improve the performance of an HMM-based cursive handwriting recognition system."International journal of Pattern Recognition and Artificial intelligence 15, no. 01 (2001): 65-90.

[13] Vinciarelli, Alessandro, and Juergen Luettin. Off-line cursive script recognition based on continuous density HMM. No. EPFL-REPORT-82562. IDIAP, 1999.

[14] Favata, John T., and Geetha Srikantan. "A multiple feature/resolution approach to handprinted digit and character recognition." International journal of imaging systems and technology 7, no. 4 (1996): 304-311.

[15] Bozinovic, Radmilo M., and Sargur N. Srihari. "Off-line cursive script word recognition." Pattern Analysis and Machine Intelligence, IEEE Transactions on 11, no. 1 (1989): 68-83.

[16] Bunke, Horst, Markus Roth, and Ernst Günter Schukat-Talamazzini. "Off-line cursive handwriting recognition using hidden Markov models." Pattern recognition 28, no. 9 (1995): 1399-1413.

[17] ARICA, NAFIZ. "An off-line character recognition system for free style handwriting." PhD diss., MIDDLE EAST TECHNICAL UNIVERSITY, 1998.

[18] Tay, Yong Haur, Pierre-Michel Lallican, Marzuki Khalid, Christian Viard-Gaudin, and S. Kneer. "An offline cursive handwritten word recognition system." In TENCON 2001. Proceedings of IEEE 
Region 10 International Conference on Electrical and Electronic Technology, vol. 2, pp. 519-524. IEEE, 2001.

[19] Gupta, Anshul, Manisha Srivastava, and Chitralekha Mahanta. "Offline handwritten character recognition using neural network." In Computer Applications and Industrial Electronics (ICCAIE), 2011 IEEE International Conference on, pp. 102-107. IEEE, 2011.

[20] Pradeep, J., E. Srinivasan, and S. Himavathi. "Neural network based recognition system integrating feature extraction and classification for English handwritten." International Journal of EngineeringTransactions B: Applications 25, no. 2 (2012): 99.

[21] Patel, D. K., T. Som, and M. K. Singh. "Improving the Recognition of Handwritten Characters using Neural Networkthrough Multiresolution Technique and Euclidean Distance Metric." International Journal of Computer Applications 45, no. 6 (2012): 38-50.

[22] Blumenstein, Michael, Brijesh Verma, and Hasan Basli. "A novel feature extraction technique for the recognition of segmented handwritten characters." In Document Analysis and Recognition, 2003. Proceedings. Seventh International Conference on, pp. 137-141. IEEE, 2003.

[23] Hallale, Sumedha B., and Geeta D. Salunke. "Twelve Directional Feature Extraction for Handwritten English Character Recognition." International Journal of Recent Technology and Engineering 2, no. 2 (2013).

[24] Choudhary, Amit, Rahul Rishi, and Savita Ahlawat. "Off-line handwritten character recognition using features extracted from binarization technique."AASRI Procedia 4 (2013): 306-312.

[25] Cruz, Rafael MO, George DC Cavalcanti, and Tsang Ing Ren. "An ensemble classifier for offline cursive character recognition using multiple feature extraction techniques." In Neural Networks (IJCNN), The 2010 International Joint Conference on, pp. 1-8. Ieee, 2010. 